\title{
Marchés financiers et développement économique : une approche historique
}

\author{
Pierre-Cyrille Hautcoeur \\ EHESS-PSE
}

Paru dans Regards croisés sur l'économie, 2008/ 1 - № 3

Toute l’ambiguïté de l'expression «marchés financiers » tient à la distance entre le concept et la réalité historique et vécue. En théorie, les marchés financiers incluent l'ensemble des moyens par lesquels des instruments financiers (des créances en première approximation) sont échangés librement, que ce soit entre un prêteur et un emprunteur (le marché primaire) ou entre détenteurs de ces créances (le marché secondaire). Ces échanges peuvent prendre des formes concrètes très variées, dans lesquelles le rôle central peut être joué par des banques, d'autres institutions financières publiques ou privées, voire des institutions non financières. Pourtant, lorsqu'on parle de marchés financiers, on pense en premier lieu à des organisations spécifiques, les «bourses de valeurs », dédiées à l’échange de titres (actions et obligations). Ce sont ces bourses, Euronext, le New-York Stock Exchange, la Bourse de Tokyo, qui constituent aujourd'hui visiblement le cœur du marché financier. Ce rôle central est renforcé par la théorisation des marchés financiers sur le modèle des bourses depuis Walras, qui a souligné la valeur de la centralisation des opérations pour la liquidité et l'obtention d'un juste prix à l'équilibre des offres et des demandes de capitaux. Même si cette vision a été relativisée au profit d'une approche plus décentralisée de la concurrence censée réaliser l'équilibre du 
marché quels qu'en soient les organisateurs, il reste que les marchés boursiers restent la référence implicite des travaux de finance tant empiriques que théoriques.

L’écart entre ces deux définitions des marchés financiers est également ressenti par tous ceux qui ont tenté de mesurer le développement financier, et avec lui l'impact du secteur financier sur le développement économique ${ }^{1}$. Dans tous les cas en effet, ils ont tenté d’inclure à la fois les marchés boursiers (mesurés par la capitalisation boursière des titres cotés) et les autres institutions financières, en particulier les banques (dont l'activité est mesurée par le total de leur bilan), quand bien même celles-ci n’agiraient pas nécessairement (que l'on pense à des banques publiques distribuant des crédits selon des critères planifiés) selon le paradigme classique du marché. Outre les grandes difficultés de mesure (les opérations financières changent dans le temps, ne sont pas toujours bien enregistrées statistiquement), une approche quantitative de la relation entre développement financier et développement économique se heurte à des problèmes de causalité réciproque non seulement difficiles à résoudre, mais peutêtre vains : sur le long terme, la question centrale est sans doute de savoir si le secteur financier s'adapte aux transformations économiques et s'il l'oriente qualitativement. Une approche historique permet ainsi sans doute moins de mesurer que de comprendre les interactions entre développements financier et économique.

Nous examinerons brièvement dans ces quelques pages d'abord comment un certain nombre de préalables idéologiques et politiques ont dû être surmontés pour rendre légaux les principaux instruments financiers et leur échange. Puis nous montrons le rôle moteur des dettes publiques dans l'émergence de marchés organisés de titres dans l'Europe moderne et la dépendance de celle-ci envers une conception libérale de l’Etat inégalement acceptée. Nous examinons par la suite les conditions qui ont présidé à l'extension de ces marchés organisés aux titres émis par les entreprises privées. Enfin, nous montrerons comment les marchés financiers, à la fois au sens large et au sens étroit, ont connu au $20^{\mathrm{e}}$ siècle recul puis renaissance.

L’existence de marchés financiers suppose en premier lieu la légalité du crédit. L’interdit religieux envers l'usure (c’est-à-dire l'intérêt) présentait dans l'Occident médiéval comme dans certains pays islamiques aujourd'hui un obstacle important, qui n’a été levé que

\footnotetext{
${ }^{1}$ Le travail pionnier est celui de Raymond W. Goldsmith, Financial structure and development, Yale U.P., 1968. Pour une synthèse récente, voir Ross Levine, « Finance and Growth », in Philippe Aghion \& Steven Durlauf, Handbook of macroeconomics, Elsevier, 2005.
} 
lentement ${ }^{2}$. Le crédit à court terme s'est épanoui grâce à l'invention de la lettre de change, qui en fournissant des services de change et de déplacement géographique des paiements masquait l'élément de crédit qui lui était pourtant consubstantiel. Le crédit à long terme a trouvé deux voies de développement: la première est la participation à l'entreprise, la commenda italienne (qui a donné la commandite française, toujours en usage) par laquelle un capitaliste apporte des capitaux à un entrepreneur en assumant une partie des risques et en échange d’une partie des bénéfices; la seconde est le contrat de rente, qui n’est spécialement grâce à une déclaration papale de 1251 - pas considéré comme un crédit (qui serait usuraire) mais comme l'échange d'un versement immédiat contre un flux de revenus soit pour la durée de vie du bénéficiaire (rente viagère), soit perpétuellement (rente perpétuelle). Il trouve son origine non dans le droit romain mais dans l'économie des monastères carolingiens, et s'ancre dans la propriété terrienne (la rente foncière) et non dans le commerce.

Ces deux instruments commencent de jouer un rôle financier important au $13^{\mathrm{e}}$ siècle, à l'apogée de l'économie médiévale qui précède la peste noire. Ils doivent pour cela surmonter un deuxième obstacle, le caractère non négociable des créances (le fait que leurs détenteurs ne peuvent pas les céder à des tiers, ou seulement avec l'accord du débiteur ou du prêteur initial). Contrats entre deux parties, les lettres de changes comme les rentes ne sont pas spontanément négociables, de même qu'un crédit bancaire ne l'est pas automatiquement aujourd'hui. Or cette négociabilité est essentielle aussi bien à la réalisation d'énormes économies par la compensation des lettres de change circulant entre places en sens inverses, que, de manière encore plus essentielle, à la simple existence de rentes quand les prêteurs trouvent - et c'est le cas général - trop risqué d’immobiliser définitivement leurs capitaux. Mais si les négociations de lettres de change comme de rentes se développent dès leur origine, ils sont durablement freinés. Pour les lettres de change, les tribunaux ne les reconnaissent qu’à partir de 1436 en Angleterre, et un peu plus tard sur le continent. Pour les rentes, les transferts sont longtemps soumis aux conditions, sévères et coûteuses, des immeubles, à la fois parce que les rentes tirent de ceux-ci leur caractère non-usuraire, parce que la séparation de la créance et du créancier choque dans une société d’ordres où la propriété (terrienne) fonde les statuts sociaux, et parce que l'Eglise perçoit bien que la possibilité d'acheter et revendre aisément les rentes équivaut à la légalisation d'un marché du crédit. C’est malgré tout bien ce qui se

\footnotetext{
${ }^{2}$ Bartolomeo Clavero, La Grâce du don. Anthropologie catholique de l'économie moderne, Albin Michel, 1996 (traduction de l'original espagnol de 1991 par J.-F. Schaub). John Munro, "The Medieval Origins of the Financial Revolution: Usury, Rentes, and Negotiability", International History Review 25:3 (2003), 505-562.
} 
produit par des transferts simplifiés offerts par certains emprunteurs, ou par l'échange de promesses de ventes qui deviennent l'équivalent de titres au porteur.

Ce sont, dès le $13^{\mathrm{e}}$ siècle, des collectivités publiques qui développent le rôle des rentes dans le marché financier. Les pionnières sont des villes libres du nord de la France, qui obtiennent de Saint Louis un statut de «corporations » les autorisant à engager leurs revenus fiscaux et même la richesse de leurs bourgeois en garantie des rentes à verser, ce qui leur donne une crédibilité inédite auprès des prêteurs. Librement consenties et transférables, elles correspondent mieux à la définition actuelle d'une dette publique vendue sur un marché que les emprunts forcés que les élites marchandes des cités italiennes s’imposent à la même époque, et qui adoptent la forme des rentes un peu plus tard. En effet, les villes picardes et flamandes empruntent plus souvent à l'extérieur qu'à l'intérieur, de sorte que leurs emprunts ne peuvent être qualifiés de simples instruments de contrôle politique des ressources fiscales internes.

Les siècles suivants voient l'extension de l'usage des rentes à des entités politiques plus importantes, donc plus puissantes et plus susceptibles de faire défaut sans laisser aux prêteurs de moyens de rétorsion autre que leur refus futur de prêter (un moyen bien faible sans capacité de coalition des prêteurs comme le montre A. Greif ${ }^{3}$ ). On l'observe dans les états de Hollande (sous souveraineté Habsbourg) en 1485 puis surtout après 1542, en France dans les émissions de l’Hôtel de Ville de Paris (qui les réalise certes pour le bénéfice du Roi, mais présente ses propres garanties) à partir de 1522 et surtout de 1553, et plus encore dès 1489 lorsque les couronnes d'Aragon et de Castille unies pour le Reconquête procèdent ensemble à l'émission de juros dont le marché à été créé par les municipalités des deux royaumes dès le $14^{\mathrm{e}}$ siècle. Le succès est général, et les taux d'émission s'abaissent souvent au denier 18 (1 pour 18 , soit 5,55\%), tandis que les montants collectés sont très importants. Des marchés organisés apparaissent à Bruges (1409), Anvers (1460), Lyon (1462) ou Amsterdam (1530). Anvers au $16^{\mathrm{e}}$ siècle et Amsterdam jusqu’à 1792 jouent un rôle de marché financier européen.

Avec la Glorieuse révolution anglaise de 1688 apparaît un nouveau modèle d'endettement public, toujours fondé sur les rentes mais s'appuyant également sur de nouvelles institutions: les grandes compagnies privilégiées. L'existence de telles corporations n'est pas en soi une nouveauté (elles jouaient déjà un grand rôle dans le commerce à grande distance). Ce qui l'est, c'est le privilège qui leur est donné comme

\footnotetext{
${ }^{3}$ Avner Greif, "Théorie des jeux et analyse historique des institutions", Annales, histoire, sciences sociales, 1998 $\mathrm{n}^{\circ} 3$ (mai-juin), pp. 597-633.
} 
créanciers monopolistes - au moins un temps - de l’Etat. La Banque d’Angleterre (créée en 1694), puis la Compagnie des Indes et la Compagnie des mers du sud, achètent en effet la dette publique et émettent elles-mêmes leurs propres titres dans le public. Elles constituent ainsi une coalition organisée des créanciers de l'Etat, ce qui rend le défaut de celui-ci difficile. La lecture positive de cet épisode ${ }^{4}$ en fait le début de la dette publique moderne, en soulignant la croissance de la dette anglaise au cours du $18^{\mathrm{e}}$ siècle, la baisse des taux d'intérêt et la diffusion des rentes dans une population beaucoup plus nombreuse. Elle y voit aussi l'une des origines de la révolution industrielle anglaise en affirmant que les emprunteurs privés purent également bénéficier d’un marché financier profond et de taux plus bas.

Une lecture plus critique montre qu'une dette publique négociable, pour des montants importants et à des taux faibles a existé ailleurs (au moins dans les trois cas précités), en particulier parce qu'une limitation par les emprunteurs des pouvoirs de l'Etat existe dans toute l'Europe occidentale, et que le régime parlementaire britannique n'en est donc pas une condition nécessaire même si elle est peut-être la plus aboutie.

Plus grave, la révolution anglaise voit se constituer une élite qui à la fois contrôle le pouvoir politique (par le Parlement) et détient la dette publique (par la Banque), selon le modèle ploutocratique que l’on avait observée dans les cités républicaines italiennes. Il n’est pas certain que les taxes indirectes développées par ces élites pour assurer le paiement des rentes constituent le meilleur encouragement au développement économique ${ }^{5}$. A cet égard, on peut s’interroger sur l'efficacité relative du modèle britannique centré sur les créanciers et du modèle d'endettement français de la même époque, dans lequel c'est l'Etat emprunteur qui domine le marché financier, utilisant son pouvoir de marché (spécialement important pour les rentes viagères, auxquelles il recourt massivement) pour emprunter à des taux relativement bas si l'on considère les risques que savent courir les prêteurs, et jouant face à ceux-ci de réductions d'intérêts unilatérales qui réduisent le coût ex-post des dettes à un niveau similaire à celui qu’obtient le plus « crédible » Etat britannique. La préférence entre les deux modèles risque de dépendre de la croyance dans la possibilité qu'un pouvoir politique agisse au nom de l’intérêt général - un concept très français il est vrai -. Quant aux retombées sur l'économie réelle, elles ne semblent pas permettre de distinguer les modalités d'endettement

\footnotetext{
${ }^{4}$ Douglass C. North; Barry R. Weingast, "Constitutions and Commitment: The Evolution of Institutions Governing Public Choice in Seventeenth-Century England", Journal of Economic History, Vol. 49, No. 4. (Dec., 1989), pp. 803-832.

${ }^{5}$ Bruce G. Carruthers., City of Capital. Politics and Markets in the English Financial Revolution, Princeton U.P., 1996.
} 
des deux pays puisque la croissance du PIB comme du commerce y est similaire jusqu’à 1789.

Quoi qu'il en soit, les vainqueurs ont toujours raison, et c'est la situation des Anglais en 1815, ce qui conduit à la diffusion européenne puis mondiale du modèle libéral de la dette publique unifiée (essentiellement une catégorie de titres homogènes), cotée sur un marché centralisé, et donc très liquide, cette dette sans risque que la théorie financière actuelle voit depuis Markovitz en composante privilégiée de tout portefeuille bien conçu Au $19^{\mathrm{e}}$ siècle, cette diffusion est l'un des moyens principaux de l'intégration financière internationale : les dettes publiques du monde entier sont cotées sur les grandes bourses européennes, Londres et Paris en premier lieu, et deviennent les instruments privilégiés des paiements ou des crédits internationaux. Cela commence par les emprunts émis par les nouveaux états latinoaméricains dès leur indépendance dans les années 1820, et finit à la veille de 1914 par les emprunts russes ou ottomans de mauvaise mémoire. L'apprentissage n’est pas toujours facile, car les emprunteurs souverains tentent - malgré les canonnières - de rester souverains, et préfèrent parfois faire défaut à soigner leur réputation malgré les suggestions de leurs prêteurs.

Ce qui fait pourtant la véritable spécificité du $19^{\mathrm{e}}$ siècle n’est pas cette convergence des pratiques de gestion libérale de la dette publique que seule l'inflation du $20^{\mathrm{e}}$ siècle remettra en question ; c'est plutôt l'émergence d'un marché centralisé pour des titres privés ${ }^{6}$. Un marché financier développé permettait déjà bien avant la Révolution le financement des agents privés. S’il fut longtemps mal observé, c'est qu'il prenait des formes depuis disparues. En l'absence de sociétés anonymes, les emprunteurs comme les prêteurs étaient des personnes privées, parfois immensément riches, souvent plus modestes. En France et dans les pays héritiers du droit romain, les notaires - détenteurs privilégiés d'informations sur les fortunes familiales du fait en particulier de leur rôle nécessaire dans les contrats de mariage - servaient d'intermédiaires financiers. Ils s’organisaient entre eux de manière à trouver des contreparties satisfaisant leurs clients, ce qui conduisait à une intégration décentralisée du marché financier ; si les notaires parisiens y jouaient un rôle plus important, ils ne formaient pas pour autant une organisation centralisée ${ }^{7}$. Si les sources nécessaires ne permettent pas de mesurer précisément cette intégration, l'importance des montants en jeu (dont l'ordre de grandeur est

\footnotetext{
${ }^{6}$ Pierre-Cyrille Hautcoeur (dir.), Le marché financier français au 19e siècle, Publications de la Sorbonne, 2007. ${ }^{7}$ Philip Hoffman, Gilles Postel-Vinay \& Jean-Laurent Rosenthal, Des marchés sans prix, éditions de l’EHESS, 2001.
} 
proche du PIB pour les crédits notariés privés à la veille de la Révolution) et l’ubiquité du crédit suggèrent qu'elle était substantielle.

Cette organisation s'effondre avec la Révolution, en particulier parce que l’hyperinflation liée aux assignats réduit presque à néant la masse des créances privées. Les banquiers prennent alors la relève des notaires. Leur action est encore moins connue, car rien ne les oblige à un enregistrement durable comparable aux archives notariales. Mais rapidement les agents privés découvrent de nouveaux instruments de financement en s’appuyant sur le marché centralisé organisé pour les titres publics. Certes, quelques entreprises avaient pu faire coter leurs titres dès le $18^{\mathrm{e}}$ siècle; mais il s'agissait de Compagnies privilégiées, étroitement surveillées par l’Etat et limitées à certaines activités.

La nouveauté du $19^{\mathrm{e}}$ siècle est triple. En premier lieu, les interdits pesant sur l'intérêt disparaissent, ce qui conduit à une grande liberté d’innovation financière qui se traduit par l'émergence des actions et obligations modernes ainsi que par la légalisation progressive d'instruments plus sophistiqués tels que les opérations à terme et optionnelles. Ensuite, la libéralisation des sociétés anonymes (entre 1850 et 1880 dans toute l’Europe) rend aisée le recours à des actionnaires extérieurs non responsables des dettes des entreprises, encore limité dans les commandites (qui requièrent au moins un commandité responsable et lui assurent la gérance). Enfin, émergent des secteurs dans lesquels la taille des entreprises et les besoins de capitaux sont tels que le recours au marché financier s'avère nécessaire, d’autant plus que l’Etat, lourdement endetté par les guerres passées, ne veut en général pas s’y engager.

La construction de nouveaux canaux permet de mettre en place les instruments et les comportements dès les années 1830, mais ce sont les chemins de fer qui bouleversent définitivement les marchés. Pendant plusieurs décennies à partir de 1840 environ (un peu plus tôt en Grande-Bretagne ou en Belgique qu'en France), des compagnies se créent et doivent avant toute recette d'exploitation construire des réseaux extrêmement coûteux, ce qui rend l'autofinancement traditionnel impossible. En outre, nombre des compagnies sont individuellement de grande taille, du fait des économies de réseau importantes dans ce secteur. Après de premières émissions d'actions, elles empruntent par obligations des montants considérables à la fois individuellement (le capital des plus grandes compagnies dépasse les 100 millions de francs, soit 1\% du PIB de 1840, et elles s’endettent bien au delà) et globalement (les émissions totalisent de l'ordre de 10\% de l'investissement national, soit 1\% du PIB, chaque année pendant plusieurs décennies).

Aucune institution financière n'est alors en mesure d'avancer durablement des montants comparables à ceux qui sont levés sur le marché, et même à court terme, les émissions 
impliquent, comme les grands emprunts publics, une mobilisation de l'ensemble du système financier (ce qui conduit d'ailleurs, évidemment, à des coûts relativement élevés). L'effet d'entraînement des chemins de fer est d'ailleurs considérable: sidérurgie et industries métallurgiques se concentrent et se développent également, en recourant parfois au marché financier ; plus encore, le système bancaire change d'échelle, avec la création de grandes banques ambitionnant de couvrir l'ensemble du territoire, et recourant au marché pour placer leurs actions et plus encore, par la suite, les émissions qu'elles organisent pour leurs clients. Si des révolutions comme celle des chemins de fer ne sont pas fréquentes, l'électricité jouera cependant un rôle similaire au début du $20^{\mathrm{e}}$ siècle par l'ampleur des investissements nécessaires et la nécessité d’un recours au marché pour les financer (même dans les cas de nationalisation).

A la veille de 1914, la capitalisation des titres cotés à la bourse de Paris représente environ trois fois le PIB (même si les titres étrangers, soit la moitié de l'ensemble, ne sont sans doute pas tous détenus en France), et, selon les estimations de l'époque, environ la moitié de la fortune nationale. Le marché boursier joue alors certainement un rôle pivot dans le marché financier français et plus généralement européen, et c’est depuis quelques grandes places financières - d'ailleurs étroitement liées entre elles - qu'est déterminé un coût du capital qui s'impose partout. Nombre de commentateurs pensent que les marchés financiers comme le capitalisme européen dans son ensemble - ont atteint leur apogée ${ }^{8}$.

Pourtant, même à cette époque, le nombre d'entreprises cotées reste faible : à peine 400 sociétés françaises cotées au marché officiel parisien en 1913, peut-être autant sur les autres marchés français, sensiblement davantage en Angleterre, mais celle-ci reste, avec la Belgique, l'exception en la matière. Les raisons de ce faible nombre sont multiples et concordantes : la plupart des entreprises recourent à l'autofinancement et ne souhaitent pas procéder à des émissions publiques qui risqueraient de diluer le contrôle - souvent familial - de leur capital ; elles préféreraient émettre des obligations, mais les Bourses exigent en général la cotation des actions pour coter des obligations; lorsque les entreprises demandent la cotation de leurs actions, c'est donc parfois par besoin fort de financement, mais le plus souvent parce que des actionnaires importants souhaitent sortir du capital (lors d'événements familiaux en particulier) et obtenir une évaluation de la valeur de leur part. La fonction de liquidité prime donc, sauf dans les secteurs que nous avons mentionnés ci-dessus, sur la fonction de financement. Par ailleurs, la demande des investisseurs pour des actions - voire des

\footnotetext{
${ }^{8}$ Voir par exemple les 7 volumes enthousiastes d’Alfred Neymarck, Finances contemporaines, F. Alcan, 1911.
} 
obligations - d'entreprises peu connues et de taille moyenne est limitée, car le risque de voir la liquidité diminuer fortement après la cotation initiale est élevé ; c’est spécialement le cas à une époque où la plupart des actions sont détenues directement par des personnes physiques, les instruments de gestion collective n'apparaissant que tardivement, timidement, et dans la seule Grande-Bretagne.

Les entreprises petites et moyennes, qui n’ont pas accès aux marchés boursiers, même régionaux, ont certes recours aux banques. Pourtant, en 1900 comme en 2000, nombre d'observateurs considèrent que trop peu de capitaux leur sont accordés si l'on tient compte de leur importance tant économique que sociale. Au nom d'externalités localisées en matière d'innovation ou de formation, on critique l'intégration centralisée des marchés financiers et on en appelle à des modes locaux de financement des districts industriels, s'appuyant sur des banques régionales, souvent mutualistes ou semi-publiques ${ }^{9}$; la force de ces banques dans les pays décentralisés comme l'Allemagne ou l'Italie, mais aussi ponctuellement en France, semble équilibrer les risques de spéculation mais aussi d'exportation excessive de capitaux. Ces revendications hostiles à une confiance aveugle aux marchés ne sont pas sans effets sur la transformation de l'articulation entre marché boursiers centralisés et marchés financiers au sens large que l'on observe au $20^{\mathrm{e}}$ siècle.

Ces marchés connaissent alors une évolution complexe. Dans un premier temps, les tendances antérieures se prolongent dans l'entre-deux-guerres en ce qui concerne les comportements des entreprises. Les années 1920 sont dans beaucoup de pays riches celles de l'élargissement des marchés boursiers à un plus grand nombre d'entreprises, d'une vive hausse des cours et d'importantes émissions liées en particulier à la vive croissance de secteurs comme l'électricité, mais aussi l'automobile ou la chimie. Néanmoins, les évolutions macroéconomiques et politiques jouent contre ce développement. L’inflation que connaissent plusieurs pays européens pendant la première guerre mondiale ruine les détenteurs d’obligations, et réduit même souvent la valeur des actions. Par ailleurs, la guerre a conduit en Europe à développer l'intervention de l'Etat dans l'économie, intervention qui dure au-delà à travers les dépenses de reconstruction et l'endettement public ou quasi-public qu'elles requièrent. La fiscalité sur les revenus du capital, quasi-nulle avant guerre, atteint des sommets dans les années 1920.

\footnotetext{
${ }^{9}$ Francesca Carnevali, Europe's Advantage. Banks and Small Firms in Britain, France, Germany and Italy since 1918, Oxford U.P., 2005.
} 
Mais le recul du marché est surtout le résultat de la crise de 1929. Considérée - avec quelque excès mais non sans raisons - comme le résultat des spéculations effrénées des années 1920, elle débouche sur une violente méfiance envers les marchés financiers ${ }^{10}$. Aux Etats-Unis, les activités bancaires et financières sont séparées strictement par le Glass Steagall Act de 1933; partout l'activité boursière recule durablement ; avant ou après la guerre, plusieurs pays (France, Grande-Bretagne, Italie entre autres) nationalisent des pans entiers de l’industrie ou des services (chemins de fer, électricité, charbonnages, banques), réduisant à peau de chagrin les capitalisations boursières. Aux investisseurs sont proposées en échange des obligations publiques ou semi-publiques qui, dans un contexte inflationniste, ne leur laisseront pas un bon souvenir. Aux Etats-Unis même, les marchés organisés jouent un moindre rôle. C’est moins les banques qui prennent leur place que des marchés financiers implicites internes aux très grandes entreprises, qui utilisent les revenus tirés de leurs activités « mûres » florissantes pour financer de nouvelles activités, selon un processus d'élargissement voire de diversification de leur activité conçu par des managers professionnels et sur lequel les actionnaires n'ont pas leur mot à dire ${ }^{11}$. Les marchés financiers continuent d'évaluer leurs actions et de financer de nouvelles entreprises, mais ils n'ont plus un rôle crucial dans l'économie. Les meilleurs observateurs prédisent alors, avec regret ou satisfaction, la fin de la domination d'un capitalisme financier qui n’a pas su satisfaire les besoins de la société ${ }^{12}$.

Le recul du rôle des marchés centralisés est particulièrement important et durable en France. On peut facilement incriminer le dirigisme français, mais en réalité un grand nombre de gouvernements depuis les années 1950 souhaitent relancer la Bourse. Ils se heurtent certes aux besoins de financement du secteur nationalisé et des priorités budgétaires du Trésor, qui conduisent celui-ci à contrôler étroitement l'allocation des crédits et à tenter d'éviter l’inflation par une étroite surveillance du « circuit » allant du crédit à la monnaie ${ }^{13}$. Mais dans les années 1960 la monnaie est stabilisée et les budgets équilibrés, et les gouvernements sont loin d’être défavorables à la Bourse comme en témoigne leur politique fiscale.

Quoi qu’il en soit, le système bancaire est réformé plus vite et efficacement que les marchés boursiers, et devient le cœur du financement de l'économie. Il est possible que la réorganisation des marchés organisés soit plus lente et plus tardive en France, alors qu’elle est

\footnotetext{
${ }^{10}$ Douglas J. Forsyth \& Ton Notermans (dir.), Regime Changes, Macroeconomic Policy and Financial Regulation in Europe from the 1930s to the 1990s, Providence \& Oxford : Berghahn Books, 1997

11 Adolph Berle \& Gardiner Means, The Modern Corporation and Private Property, Macmillan, 1933.

${ }^{12}$ Karl Polanyi, La grande transformation, Gallimard, 1983 (éd. originale anglaise en 1944) ; Joseph Schumpeter, Capitalisme, socialisme et démocratie, Payot, 1983 (éd. originale anglaise en 1942).

${ }^{13}$ Laure Quennouëlle-Corre, La direction du Trésor, 1947-1967. L'Etat-banquier et la croissance, Comité pour l’histoire économique et financière de la France, 2000.
} 
plus continue et s'accélère dans les années 1970 et 1980 dans les pays anglo-saxons. Au $19^{\mathrm{e}}$ siècle, l'organisation de la bourse de Paris lui permettait d'assurer un équilibre entre dynamisme et sécurité peut-être plus satisfaisant que celle de son homologue londonienne. En effet, elle combinait un marché officiel (organisé par la Compagnie des agents de change) et un marché officieux (la coulisse) présentant des combinaisons de garantie des opérations et de liquidité complémentaires, et donc à même d'attirer des catégories différentes aussi bien d'émetteurs que d'investisseurs. Cet ensemble parisien était complété par quelques marchés provinciaux, dont un marché important à Lyon pour les titres industriels (électricité, chimie, tramways). Les deux guerres mondiales affaiblirent fortement la coulisse (ruinée par la première, décimée de ses nombreux intermédiaires juifs durant la seconde), et conduisirent à un renforcement du monopole des agents de change (qui obtinrent l'absorption des coulissiers comme des bourses de province), dont la prudence tendit désormais vers le pur conservatisme, évitant toute véritable réforme pendant les Trente glorieuses.

Ce n'est que sous l'impulsion du gouvernement socialiste de P. Bérégovoy - motivé sans doute en partie par la nécessité d’un marché efficace pour financer une dette publique en forte croissance - que la Bourse de Paris connaît à partir de 1984 sa grande restructuration. Nouveaux instruments (futures, options, warrants, produits indexés, etc...), nouveaux marchés, nouvelle organisation (avec la disparition des agents de change, la création du marché continu, puis la transformation de la Bourse en société anonyme), nouvelles ambitions (avec le développement international d'Euronext), la Bourse reprend une place importante dans l'économie française comme dans les autres pays développés, dans une convergence qui semble indiquer la fin des espoirs d'une diversité des formes de capitalisme ${ }^{14}$. La logique financière de marché reprend aussi ses droits dans l'activité des banques (qui titrisent par exemple certains de leurs actifs, ou mesurent leurs risques à partir de modèles inspirés de la finance de marché) et dans le contrôle des entreprises, soumises à l'obligation de convaincre au jour le jour de la pertinence de leurs décisions sous peine de remise en cause de leur direction. L’intégration financière internationale (le contrôle des changes disparaît à partir de 1986), renforcée par l'adoption de l'euro, semblent ôter l'essentiel de leurs pouvoirs financiers aux gouvernements, comme au début du $20^{\mathrm{e}}$ siècle. La confiance dans les marchés

\footnotetext{
${ }^{14}$ Peter A. Hall \& David Soskice (dir.) Varieties of capitalism: the institutional foundations of comparative advantage, Oxford university Press, 2001
} 
financiers connaît un renouveau qui s’appuie sur la théorie financière et renforce la révolution libérale ${ }^{15}$.

Pour autant, les incertitudes sur l'efficacité des marchés financiers ne sont pas résolues : les comportements mimétiques et à courte vue provoquant des bulles et des crises semblent bien avérés (la crise boursière de 2001 est la plus aiguë de l’histoire, quoique pas la plus profonde), tout comme les asymétries d'information, avec non seulement les délits d'initiés mais les risques d'erreurs d'allocation du capital ainsi que d’illiquidité du système bancaire qu'elles comportent. Aujourd'hui comme autrefois, les innovations financières comportent plus que les innovations technologiques - de fortes dimensions redistributives, comme en témoigne tout récemment la vogue des subprime (les crédits hypothécaires pourris construits sur le modèle des junk bonds des années 1980), et conduisent souvent à l'effondrement après l'engouement ${ }^{16}$. Les inégalités entre emprunteurs comme entre investisseurs, et les profits faramineux de certains intermédiaires appellent à de meilleures régulations assurant équité et concurrence. Certains jugent que des institutions financières sans but lucratif, sur le modèle des banques mutualistes devraient être de nouveau développées au profit des PME et des épargnants prudents; d'autres que le retour des grands capitalistes individuels appelle celui de la responsabilité illimitée. En tout état de cause, le capitalisme financier, malgré son âge, semble bien n'avoir pas encore toute sa raison, et l'effort permanent de ses thuriféraires pour convaincre de sa neutralité et de son efficacité spontanées démontrent, comme son histoire, la dimension inévitablement politique de sa régulation.

\footnotetext{
${ }^{15}$ Michael C. Jensen (Theory of the Firm: Governance, Residual Claims, and Organizational Forms, Harvard University Press, 2000) synthétise cette révolution théorique,qui est racontée par Peter Bernstein, Capital Ideas : the improbable origins of modern Wall Street, New-York, Free Press, 1991.

${ }^{16}$ Mitchel Abolafia, Making Markets : Opportunism and Restraint on Wall Street, Harvard University Press, 2001.
} 